\title{
Bone mineral density status in urolithiasis patients with vitamin D inadequacy followed at a tertiary stone centre
}

\author{
Mohamed A. Elkoushy, MD, MSc, PhD; ${ }^{* *}$ Mazen Jundi, MD; ${ }^{*}$ Terence T.N. Lee, MD; \\ Sero Andonian, MD, MSc, FRCSC, FACS*
}

"Division of Urology, Department of Surgery, McGill University Health Centre, McGill University, Montreal, QC; 'Department of Urology, Suez Canal University, Ismailia, Egypt

Cite as: Can Urol Assoc J 2014;8(9-10):323-8. http://dx.doi.org/10.5489/cuaj.2055 Published online October 13, 2014.

\section{Abstract}

Introduction: We assessed abnormalities in bone mineral density (BMD) and the risk of hip and major osteoporotic fractures in urolithiasis patients with vitamin D inadequacy (VDI) followed at a tertiary stone centre.

Methods: Stone-free patients with VDI were invited to undergo dual-energy x-ray absorptiometry (DXA) scans to assess for BMD abnormalities at the femoral neck and lumbar spine. The World Health Organization's validated Fracture Risk Assessment Tool (FRAX) was used to calculate the risk of hip and major osteoporotic fractures within 10 years. Patients with primary hyperparathyroidism or hypercalcemia were excluded.

Results: In total, 50 consecutive patients were included between June 2011 and August 2012, including 26 (52\%) males. The median age was 51 years and the median 25-hydroxyl vitamin D $(25[\mathrm{OH}]$ D) was $18.8 \mathrm{ng} / \mathrm{mL}$. Thirty patients $(60 \%)$ had abnormal T-scores on DXA studies. This decreased to 22 (44\%) when age-matched Z-scores were used; $36 \%$ had osteopenia and $8 \%$ had osteoporosis. Femoral neck and lumbar spines were affected in $24 \%$ and $32 \%$ of patients, respectively. Recurrent stone-formers had significantly lower BMD when compared with first-time stone formers. Median serum $25(\mathrm{OH}) \mathrm{D}$ was comparable between patients with normal and abnormal DXA scans (18.6 vs. $18.8 \mathrm{ng} / \mathrm{mL} ; p=0.91)$. Five patients $(10 \%)$ were at high risk $(\geq 3 \%)$ of hip fractures within 10 years.

Conclusion: A high prevalence of abnormal DXA scans was found in urolithiasis patients with VDI, including 5 patients (10\%) at high risk of hip fractures. Future studies need to assess the economic impact of obtaining DXA scans on urolithiasis patients with VDI, especially in recurrent stone-formers.

\section{Introduction}

Osteoporosis is the most common bone disease globally. ${ }^{1}$ By 2020, it is estimated that 14 million individuals over 50 years will develop osteoporosis and another 47 million will have osteopenia in the United States alone. ${ }^{1}$ Furthermore, osteoporosis is the most common cause of fractures, accounting for 1.5 million yearly fractures in the United States. ${ }^{1}$ In addition to the associated physical and mental morbidities of osteoporotic fractures to individuals, it is estimated that 12 to 18 billion dollars are spent per year as annual direct care costs to treat such osteoporotic fractures; this cost exceeds costs related to heart disease, stroke and breast cancer. ${ }^{2}$ Therefore, osteoporosis has an impact on global health and it is important to identify patients at risk of osteoporotic fractures and institute appropriate preventive measures.

Several population-based cohort studies have examined the relationship of urolithiasis and osteoporosis. ${ }^{3-5}$ However, most of the studies were conducted before the introduction of Z-scores based on the dual-energy $\mathrm{x}$-ray absorptiometry (DXA) scan, which is the current gold standard to evaluate osteoporosis and assess fracture risk according to the World Health Organization (WHO). ${ }^{6}$ In addition, none of these studies examined the role of vitamin D inadequacy (VDI) in these patients. Vitamin D plays a vital role in calcium homeostasis and bone health. ${ }^{7}$ Recent studies have confirmed a worldwide trend in VDI, ${ }^{8-11}$ including $64 \%$ to $90 \%$ of osteoporotic patients. ${ }^{11-14}$ In addition, $80 \%$ of patients presenting with urolithiasis had VDI. ${ }^{15}$ Therefore, it is important to assess vitamin D status in urolithiasis patients who are at higher risk of developing osteoporosis.

Since most osteoporotic fractures occur in patients with osteopenia rather than osteoporosis, the WHO has come up with the Fracture Risk Assessment Tool (FRAX), which is an internationally validated assessment tool that calculates 10-year probabilities of hip and major osteoporotic fractures. ${ }^{1}$ Since none of the previous studies had calculated the FRAX score, the aim of the present study was to assess abnormalities in BMD studies in urolithiasis patients with VDI in addition to identify patients who are at high risk for hip and major osteoporotic fractures. 
Elkoushy et al.

\section{Methods}

Serum vitamin 25-hydroxyl vitamin D $(25[\mathrm{OH}] \mathrm{D})$ and parathyroid hormone (PTH) levels were routinely obtained as part of a full metabolic stone workup of all patients presenting to our tertiary stone clinic. VDI was defined as a $25(\mathrm{OH}) \mathrm{D}$ level $<30 \mathrm{ng} / \mathrm{mL} .{ }^{11}$ Stone-free patients with VDI were offered DXA scans to assess for BMD abnormalities. All VDI patients who underwent DXA scans between June 2011 and August 2012 were included in the study. Parathyroid scans and neck ultrasonography were performed in patients with elevated PTH to differentiate between primary and secondary hyperparathyroidism. Patients with primary hyperparathyroidism or hypercalcemia were excluded.

BMD was measured by DXA scans at the lumbar spines $\left(\mathrm{L}_{2-4}\right)$ and femoral neck utilizing Lunar DPX scanner and Lunar Ortho software (Lunar Corporation, Madison, WI. Results were expressed as $\mathrm{g} / \mathrm{cm}^{2}$ (Fig. 1). The combined BMD was calculated as the sum of scores calculated for both the femoral neck and lumbar spines.

T-score represents the number of standard deviations below the average for a young (20-29 years old) Caucasian adult female at peak bone density. Therefore, T-score varies depending on bone densitometer, skeletal site, age, race and gender. In contrast, Z-score is the number of standard deviations below a reference group consisting of people of the same age, race, gender and weight. Therefore, Z-scores are more reliable. The WHO criteria were used to define patients with abnormal BMD (Table 1).

FRAX is an internationally validated tool from the WHO. ${ }^{16}$ This public freeware calculates the 10-year risk of hip fractures and the 10-year risk of a major osteoporotic fracture (spine, forearm, hip or shoulder fracture). ${ }^{1}$ It is based on individual risk factors (age, gender, ethnicity, body mass

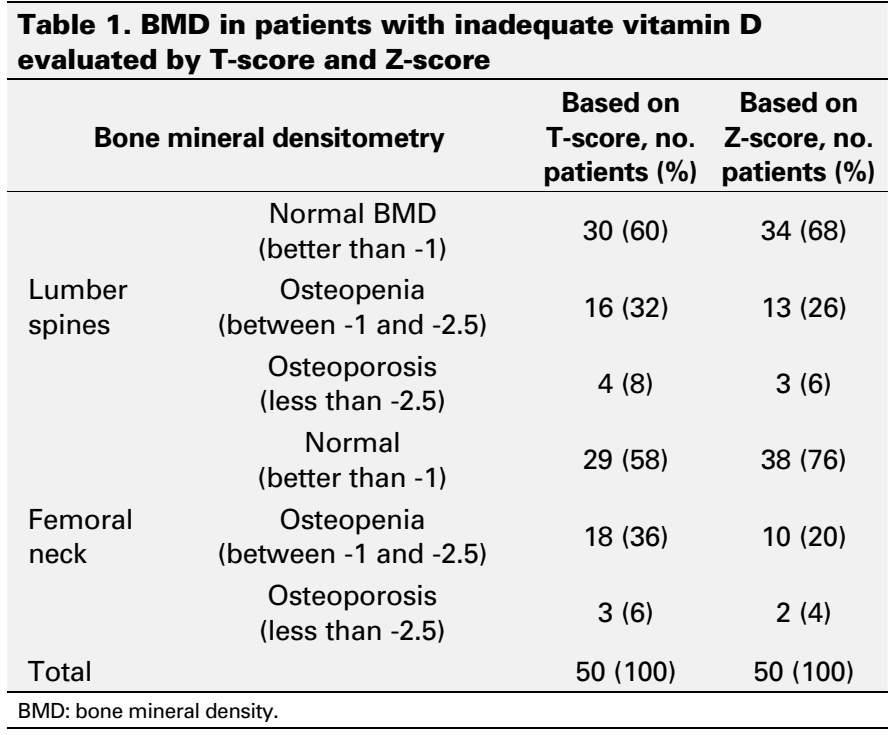

index [BMI], previous fracture, parent hip fracture, active smoker, secondary osteoporosis due to excessive alcohol consumption [ $>3$ drinks/day], glucocorticoids or rheumatoid arthritis), as well as BMD of the femoral neck. According to the National Osteoporosis Foundation, based on the FRAX score, patients in the high-risk groups with $\geq 3 \%$ hip fracture risk or with $\geq 20 \%$ risk of a major osteoporotic fracture within 10 years are recommended to be treated pharmacologically. ${ }^{1}$

\section{Statistical analyses}

Data analysis was done using the commercially available Statistical Package for Social Sciences for Windows, version 20 (SPSS, Chicago, IL). Descriptive data were presented in terms of numbers, percentages, medians $\left(25^{\text {th }}\right.$ to 75 th percentiles), and means with 95\% confidence intervals (Cl). Mann Whitney- $U$ and Kruskal Wallis- $\mathrm{H}$ tests were used to compare continuous variables, while Fisher exact test was used to compare categorical variables. A two-sided level of significance under 0.05 was adopted. Pearson correlation was used to detect relationship between BMD and each of the serum and urinary parameters.

\section{Results}

In total, we included 50 consecutive urolithiasis patients with VDI who underwent DXA scans. There were 26 (52\%) males and $28(56 \%)$ recurrent stone-formers. Twenty-one patients $(42 \%)$ had family history of urolithiasis. The median age (25th-75th percentiles) was 51 (42.8-62.0), median BMI was $28.4 \mathrm{~kg} / \mathrm{m}^{2}(24.8-30.5)$ and median serum vitamin D was $18.8 \mathrm{ng} / \mathrm{mL}$ (13.6- 22.1). The median total and ionized normalized serum calcium levels were $9.32 \mathrm{mg} / \mathrm{dL}$ (8.96$9.44)$ and $4.8 \mathrm{mg} / \mathrm{dL}$ (4.60- 4.92), respectively. The median intact PTH was $45.2 \mathrm{pg} / \mathrm{mL}$ (44.8- 63.6).

Out of the 50 patients, $30(60 \%)$ had abnormal T-scores on DXA studies. Of these 30, 24 (48\%) had osteopenia and $6(12 \%)$ had osteoporosis in the femoral neck and/or lumbar spines. When using Z-scores, 22 patients (44\%) had abnormal DXA studies, of which 18 (36\%) had osteopenia and 4 $(8 \%)$ had osteoporosis in the femoral neck and/or lumbar spine. Twelve (24\%) patients had abnormal BMDs in the femoral neck and $16(32 \%)$ patients had abnormal BMDs in the lumbar spine, including a patient with vertebral fractures constituting severe osteoporosis (Table 1). Compared with patients with single stone episode, recurrent stone-formers had significantly lower BMD at lumbar spines, femoral neck and combined locations (1.21 vs. $1.04 \mathrm{gm} / \mathrm{cm}^{2}, p<0.001$ ), (0.98 vs. $\left.0.86 \mathrm{gm} / \mathrm{cm}^{2}, p=0.01\right)$ and (2.19 vs. $1.88 \mathrm{gm} / \mathrm{cm}^{2}$, $p<0.001)$, respectively.

Compared with patients with normal BMD, patients with abnormal Z-sores were significantly younger ( 57.5 vs. 50 , 


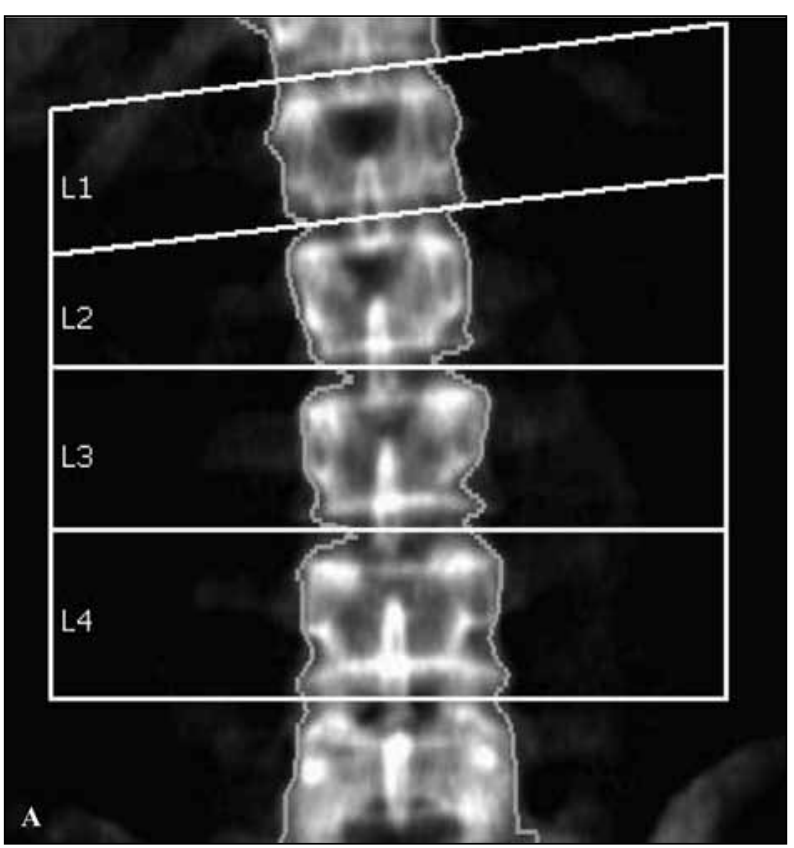

Fig. 1a. Abnormal bone densitometry of the lumbar spines $\left(\mathrm{L}_{2-4}\right)$ and femoral neck. Osteoporotic changes in $\mathrm{L}_{2-4}$ of a 50 -year-old male (bone mineral density $=0.85 \mathrm{~g} / \mathrm{cm}^{2}, \mathrm{~T}$-score $=-3.3, \mathrm{Z}$-score $=-3.1$ ).

$p=0.02)$, more males $(39.3 \%$ vs. $72.7 \%, p=0.02)$ and had significantly lower BMI (30.1 vs. $\left.25.2 \mathrm{~kg} / \mathrm{m}^{2}, p<0.001\right)$ (Table 2). However, median serum vitamin D was not significantly different between patients with normal and abnormal DXA scans (Table 2). Similarly, median serum vitamin D

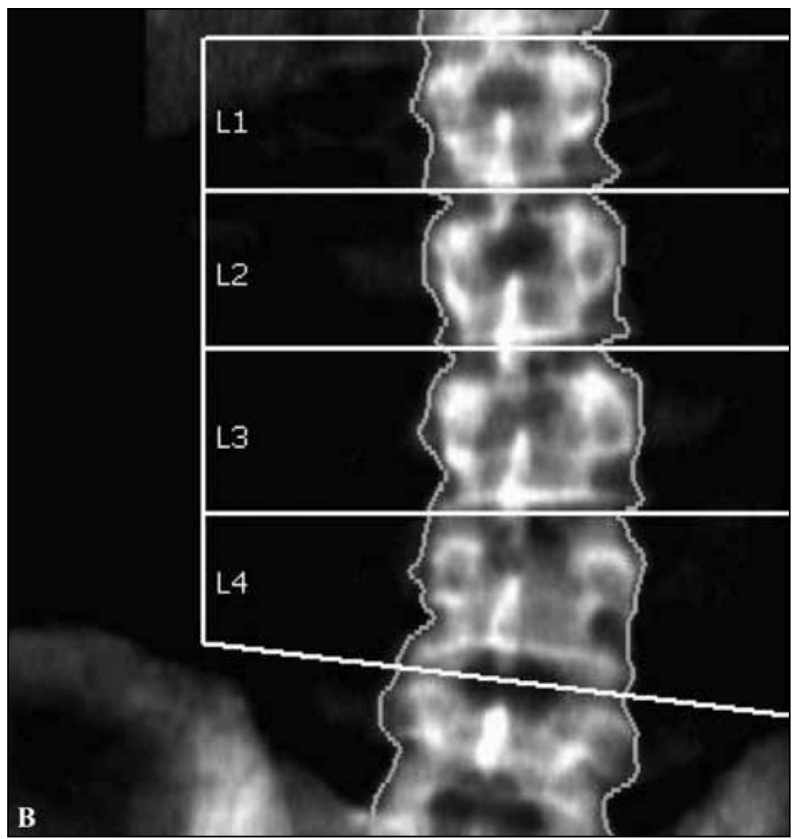

Fig. 1b. Abnormal bone densitometry of the lumbar spines $\left(\mathrm{L}_{2-4}\right)$ and femoral neck. Osteopenic changes in $\mathrm{L}_{2-4}$ of a 50 -year-old male (bone mineral density $=1.09 \mathrm{~g} / \mathrm{cm}^{2}$, T-score $=-1.3$, Z-score $=-1.1$. . did not significantly differ between osteopenics and osteoporotics (17.8 vs. $15.6 \mathrm{ng} / \mathrm{mL}, p=0.64$ ). In addition, there were no significant differences between patients with normal and abnormal Z-scores in terms of metabolic stone workup, including serum and 24-hour urinary parameters (Table 2).

However, the combined BMD significantly correlated with serum ionized normalized calcium levels $(r=0.37$, $p=0.02$ ) (Fig. 2). Otherwise, there was no correlation of BMD with other metabolic stone workup parameters. In terms of FRAX calculations, patients with normal and abnormal Z-scores had comparable risk of major osteoporotic and hip fractures fractures within 10 years (Table 2). However, 5 patients $(10 \%)$ were at high risk $(\geq 3 \%)$ of hip fractures within 10 years.

There was no significant difference between patients with normal and abnormal Z-scores supplementing with oral calcium $(21.4 \%$ vs. $36.4 \%, p=0.25)$ and/or vitamin $\mathrm{D}(50 \%$ vs. $54.5 \% ; p=0.75)$. Furthermore, compared to those with normal Z-scores, patients with abnormal Z-scores had a comparable median (range) daily calcium dose (0 [0-1000] vs. 0 [0-1890] $\mathrm{mg}, p=0.22$ ) and a median daily vitamin D dose (200 [0-2000] vs. 257 [0- 2200] IU, $p=0.29)$.

There were 41 (82\%) stone analyses available; 36 (87.8\%) were calcium-containing stones (calcium oxalate, phosphate and/or carbonate). Of interest, 3 out of 5 uric acid stone formers $(60 \%)$ had abnormal Z-scores, including one osteoporotic patient.

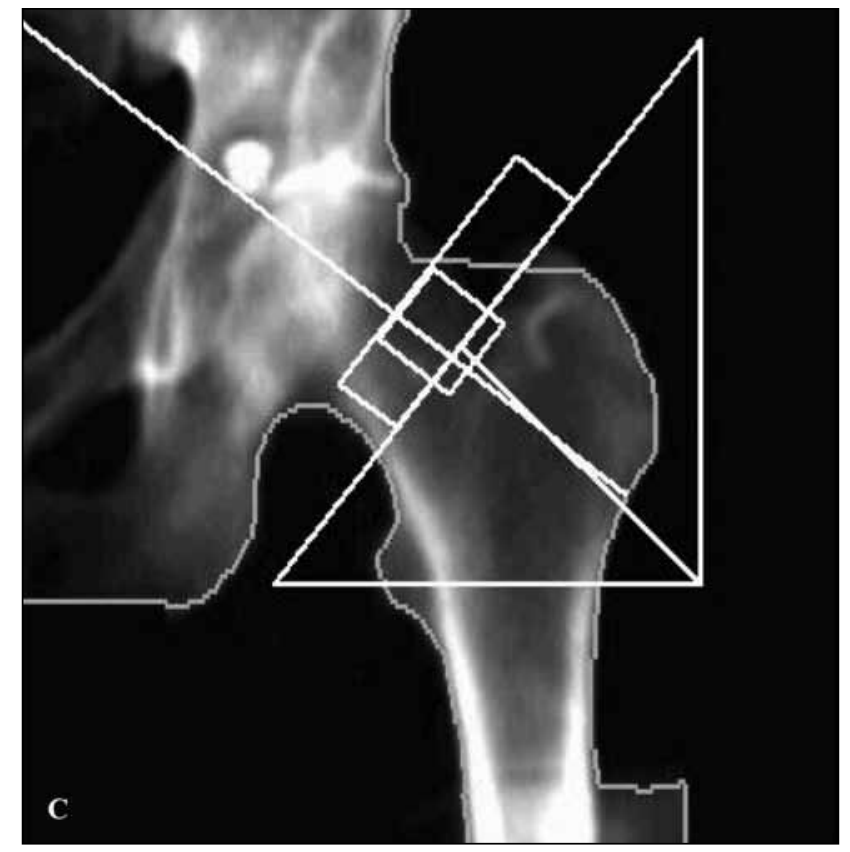

Fig. 1c. Abnormal bone densitometry of the lumbar spines $\left(\mathrm{L}_{2-4}\right)$ and femoral neck. Osteopenic changes in the femoral neck of a 52 -year-old male (bone mineral density $=0.66 \mathrm{~g} / \mathrm{cm}^{2}, T$-score $=-3.4$, Z-score $=-2.4$ ). 
Elkoushy et al.

\begin{tabular}{|c|c|c|c|c|}
\hline Variable & & Normal Z-score $(\mathrm{n}=\mathbf{2 8}$ ) & Abnormal Z-score ( $n=22)$ & $p$ value \\
\hline Median age (years) & & $57.5(48-63)$ & $50(39-65)$ & 0.02 \\
\hline Male gender & & $11(39.3 \%)$ & $16(72.7 \%)$ & 0.02 \\
\hline Median BMI (kg/m²) & & $30.1(26.2-36.4)$ & $25.2(23.0-27.9)$ & $<0.001$ \\
\hline \multirow[t]{3}{*}{$\operatorname{BMD}\left(\mathrm{g} / \mathrm{cm}^{2}\right)$} & Lumbar spines & $1.23(1.13-1.35)$ & $1.04(0.93-1.10)$ & $<0.001$ \\
\hline & Femoral neck & $0.97(0.89-1.06)$ & $0.89(0.86-1.02)$ & 0.02 \\
\hline & Combined & $2.21(1.94-2.41)$ & $1.98(1.84-2.06)$ & $<0.001$ \\
\hline \multirow[t]{2}{*}{ FRAX score } & Major osteoporotic fractures within 10 years & $4.2 \%(2.8-6.5)$ & $4.5 \%(2.6-5.0)$ & 0.37 \\
\hline & Hip fractures within 10 -years & $0.40 \%(0.20-1.10)$ & $0.45 \%(0.15-0.75)$ & 0.30 \\
\hline \multirow[t]{6}{*}{ Serum parameters } & Vitamin 25(OH)D (ng/mL) & $18.6(16.2-21.9)$ & $18.8(14.8-22.8)$ & 0.53 \\
\hline & Total calcium (mg/dL) & $9.3(9-9.5)$ & $9.4(9-9.5)$ & 0.30 \\
\hline & lonized $\mathrm{Ca}++(\mathrm{mg} / \mathrm{dL})$ & $4.8(4.6-5)$ & $4.8(4.6-4.9)$ & 0.44 \\
\hline & PTH intact $(\mathrm{pg} / \mathrm{mL})$ & $54.2(47.5-62.4)$ & $62(40.9-74.5)$ & 0.48 \\
\hline & Creatinine (mg/dL) & $0.80(0.64-1.0)$ & $0.83(0.72-1.0)$ & 0.60 \\
\hline & Uric acid (mg/L) & $6.6(4.2-7.2)$ & $5.6(5.1-7.1)$ & 0.71 \\
\hline \multirow[t]{8}{*}{ 24-hour urine collections } & Calcium (mg/day) & $212(144-308)$ & $164(128-256)$ & 0.44 \\
\hline & Phosphorous (g/day) & 752 (595-934) & 752 (570-848) & 0.78 \\
\hline & Citrate (mg/day) & $518(422-768)$ & $480(768-787)$ & 0.84 \\
\hline & Oxalate (mg/day) & $33.5(28.7-40.2)$ & $33.8(22.1-46.8)$ & 0.96 \\
\hline & Magnesium (mg/day) & $105(83-141)$ & $88(66-117)$ & 0.19 \\
\hline & Sodium (g/day) & $3.9(2.9-4.7)$ & $3.5(2.9-4.3)$ & 0.64 \\
\hline & Creatinine (g/day) & $1.29(1.18-1.68)$ & $1.47(1.23-1.63)$ & 0.20 \\
\hline & Uric acid (mg/day) & $50(44-62)$ & $59(42-67)$ & 0.18 \\
\hline
\end{tabular}

Values are given as medians (25th to 75th percentiles) or numbers (\%). 25(OH)D: 25-hydroxyl vitamin D; PTH: parathyroid hormone; FRAX: fracture risk assessment system; BMI: body mass index; BMD: bone mineral density.

\section{Discussion}

The pathophysiological relationship of BMD and kidney stones is poorly understood and involves the interplay of hormonal, genetic and environmental factors. In the present

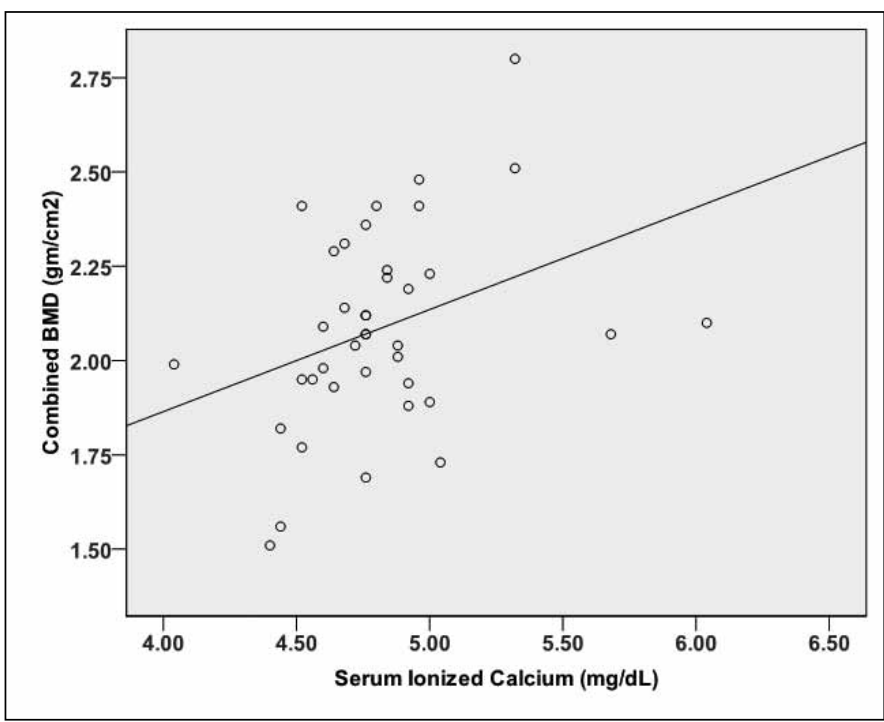

Fig. 2. Correlation of combined bone mineral density with serum ionized calcium. $\mathrm{r}=0.37 ; p=0.02$. study, the high prevalence of patients followed with urolithiasis and VDI had abnormal BMD on DXA scans. While 30 patients $(60 \%)$ had abnormal T-scores on DXA scans, this decreased to $22(44 \%)$ when age-matched Z-scores were used.

Most of the DXA scan abnormalities were in the form of osteopenia (18 patients, 36\%) and most affected the lumbar spine (16 patients, 32\%). In fact, there was 1 patient with vertebral fractures thus meeting the criteria for severe osteoporosis. Furthermore, patients with recurrent urolithiasis had significantly lower BMD when compared with firsttime stone formers. While previous studies alluded to the association between urolithiasis and low BMD, this is the first study calculating Z-scores and FRAX scores and identifying patients at high risk for hip fractures within 10 years.

The findings in the present study are supported by previous cross-sectional and longitudinal studies. For example, in the NHANES III (National Health and Nutrition Examination Survey) cross-sectional study, men with a history of urolithiasis had significantly lower femoral neck BMD than men without a history of urolithiasis. ${ }^{5}$ Whereas the NHANES III study relied on patient questionnaires to determine previous history of urolithiasis (recall bias), in the present study patients presenting with urolithiasis to a tertiary stone centre were confirmed to have urolithiasis by imaging stud- 
ies. Another limitation of the NHANES study is the lack of BMD measurement at the lumbar spine. In the longitudinal study of 624 Rochester residents presenting with urolithiasis between 1950 and 1974, there were 4 times more vertebral fractures when compared with the general population of Rochester, MN (standardized mortality ratio 3.9; 95\% Cl 3.0-4.9). ${ }^{4}$ Although this study lacked DXA scans, it confirmed the findings in the present study where there were more DXA scan abnormalities in the lumbar spines than in the femoral neck.

Previous prospective studies examining rates of osteopenia and osteoporosis concentrated on hypercalciuric and idiopathic renal calcium stone formers. ${ }^{17,18}$ Using Z-scores for men presenting with idiopathic calcium stone formers, Trinchieri and colleagues found the rates of osteopenia and osteoporosis at $48 \%$ and $6 \%$, respectively. ${ }^{17}$ Similarly, Caudarella and colleagues found 54\% osteopenia and $14 \%$ osteoporosis using qualitative ultrasound to calculate T-scores. ${ }^{18}$ While the present study confirmed these rates, it included all patients presenting with urolithiasis and not just patients with calcium stones. In fact 3 out of 5 uric acid stone formers $(60 \%)$ had abnormal Z-scores, including one osteoporotic patient. Therefore, the rates of osteopenia and osteoporosis seen here apply to all comers with urolithiasis.

There have been several theories as to why patients with urolithiasis are at an increased risk of low BMD. Some authors have hypothesized that the same dietary habits (i.e., high animal protein) that increase the risk for urolithiasis also create a slight degree of metabolic acidosis leading to a reduction of bone mass. ${ }^{18}$ In fact, treatment with alkalinizing agents, such as potassium citrate, for 1 year has been shown to significantly increase BMD at the vertebral spine, femoral neck and total hip in postmenopausal woman with osteopenia. ${ }^{19}$ Another hypothesis is a defect in vitamin D regulation with either high circulating levels of 1,25 vitamin $\mathrm{D}$ or increased bone tissue sensitivity to vitamin D. ${ }^{20}$ In the present study, vitamin D stores and VDI were assessed by measuring serum $25(\mathrm{OH})$ vitamin D levels. There was no significant difference in median serum VD levels between patients with normal and abnormal Z-scores (18.6 vs. $18.8 \mathrm{ng} / \mathrm{mL}, p=0.53)$. It is possible that circulating levels of 1,25 vitamin D might be different between the groups. Yet others have hypothesized that calcium restriction advice in the past may have contributed to the negative calcium balance since idiopathic renal calcium stone-formers with low BMD have significantly lower calcium intake when compared with calcium stone-formers with normal BMD. ${ }^{17-22}$ Recently, evidence from the NHANES III study showed that in men with history of urolithiasis, milk consumption was associated more strongly with femoral neck BMD than for men without history of urolithiasis. ${ }^{5}$

While patients with osteoporosis are at higher risk of fractures, most $(82 \%)$ fractures occur in patients with oste- openia. ${ }^{23}$ Therefore, the FRAX scoring system was adopted by the WHO and International Osteoporosis Foundation to identify and treat patients at high risk of osteoporotic fractures. ${ }^{6}$ In the present study, 5 patients $(10 \%)$ were found to be at high risk ( $\geq 3 \%$ ) of hip fractures within 10 years and were referred to bone metabolism clinic for pharmacological treatment. Although the FRAX scoring system is useful in identifying high-risk patients, it is not without its limitations. The first limitation is that it is based on the femoral neck BMD from the NHANES III database using 20- to 29-year-old female Caucasians as a reference group. ${ }^{6}$ However, abnormal BMD are seen more often in the lumbar spine than in the femoral neck. Therefore, future studies need to consider using a modified FRAX scoring system using the lumbar spine BMD to identify urolithiasis patients at high risk for vertebral fractures.

The main limitation of the current cohort is its relatively small sample size. Therefore, the lack of significant difference between patients with normal and abnormal Z-scores could be due to statistical type II error. Another limitation is the lack of dietary assessment of these patients. Nevertheless, this is the first study to report abnormal bone mineral densities based on Z-scores and assess risk of hip and major osteoporotic fractures within 10 years in patients presenting with urolithiasis and VDI. In particular, the present study identified 5 patients $(10 \%)$ who were at high risk for hip fractures. Some authors have gone as far as recommending obtaining DXA scans for all patients presenting with urolithiasis and treating patients with low BMD. ${ }^{24}$ Future studies need to examine the economic impact of this recommendation prior to incorporation into clinical practice.

\section{Conclusion}

A high prevalence of abnormal DXA scans was found in patients presenting with urolithiasis and VDI and $10 \%$ of these patients are at high risk for hip fractures within 10 years. Future studies need to assess the economic impact of obtaining DXA scans on urolithiasis patients with VDI, especially in recurrent stone-formers.

Acknowledgements: This work was supported in part by the Canadian Urological Association Scholarship Foundation and the Montreal General Hospital Foundation Awards to Sero Andonian.

Competing interests: Dr. Elkoushy, Dr. Jundi, Dr. Lee and Dr. Andonian all declare no competing financial or personal interests.

This paper has been peer-reviewed. 
Elkoushy et al.

\section{References}

1. Shuler FD, Conjeski J, Kendall D, et al. Understanding the burden of osteoporosis and use of the World Health Organization FRAX. Orthopedics 2012;35:798-805. http://dx.doi.org/10.3928/0147744720120822-12

2. Assessment of fracture risk and its application to screening for postmenopausal osteoporosis: Report of a WHO Study Group. WHO Technical Report Series 1994;843:1-129.

3. Keller JJ, Lin CC, Kang JH, et al. Association between osteoporosis and urinary calculus: Evidence from a population-based study. Osteoporos Int 2012;24:651-7. http://dx.doi.org/10.1007/s00198-0122019-5

4. Melton $\mathrm{L} \mathrm{III}$, Crowson CS, Khosla S, et al. Fracture risk among patients with urolithiasis: A population-based cohort study. Kidney Int 1998;53:459-64. http://dx.doi.org/10.1046/i.1523-1755.1998.00779.x

5. Lauderdale DS, Thisted RA, Wen $M$, et al. Bone mineral density and fracture among prevalent kidney stone cases in the Third National Health and Nutrition Examination Survey. J Bone Miner Res 2001;16:1893-8. http://dx.doi.org/10.1359/jbmr.2001.16.10.1893

6. McCloskey E. FRAX Identifying people at high risk of fracture: WHO Fracture Risk Assessment Tool, a new clinical tool for informed treatment decisions. Nyon, Switzerland: International Osteoporosis Foundation; 2009.

7. Rosen CJ, Morrison A, Zhou H, et al. Elderly women in northern New England exhibit seasonal changes in bone mineral density and calciotropic hormones. Bone Miner 1994;25:83-92. http://dx.doi. org/10.1016/50169-6009(08)80250-4

8. Mithal A, Wahl DA, Bonjour JP, et al. Global vitamin D status and determinants of hypovitaminosis $D$. Osteoporos Int 2009;20:1807-20. http://dx.doi.org/10.1007/s00198-009-0954-6

9. Kuchuk NO, van Schoor NM, Pluijm SM, et al. Vitamin D status, parathyroid function, bone turnover, and BMD in postmenopausal women with osteoporosis: Global perspective. J Bone Miner Res 2009;24:693701. http://dx.doi.org/10.1359/ibmr.081209

10. Adams JS, Hewison M. Update in vitamin D. J Clin Endocrinol Metab 2010;95:471-8. http://dx.doi. org/10.1210/ic.2009-1773

11. Holick MF: Vitamin D deficiency. N Engl J Med 2007;357:266-81. http://dx.doi.org/10.1056/ NEJMra070553

12. Guardia $G$, Parikh N, Eskridge $T$, et al. Prevalence of vitamin D depletion among subjects seeking advice on osteoporosis: A five-year cross-sectional study with public health implications. Osteoporos Int 2008; 19:139. http://dx.doi.org/10.1007/s00198-007-0456-3
13. Lips $P$, Hosking $D$, Lippuner $K$, et al. The prevalence of vitamin $D$ inadequacy amongst women with osteoporosis: An international epidemiological investigation. J Intern Med 2006;260:245-54. http:// dx.doi.org/10.1111/i.1365-2796.2006.01685.x

14. De Cock C, Bruyere 0 , Collefte J, et al. Vitamin D inadequacy in French osteoporotic and osteopenic women. Joint Bone Spine 2008;75:567-72. http://dx.doi.org/10.1016/i.jbspin.2007.10.012

15. Elkoushy MA, Sabbagh R, Unikowsky B, et al. Prevalence and metabolic abnormalities of vitamin D deficient patients presenting with urolithiasis. Urology 2012;79:781-5. http://dx.doi.org/10.1016/i. urology.2011.09.004

16. FRAX WHO Fracture Risk Assessment Tool. http://www.shef.ac.uk/FraX. Accessed September 16, 2014.

17. Tranchieri A, Nespoli R, Ostini F, et al. A study of dietary calcium and other nutrients in idiopathic calcium stone formers with low bone mineral content. J Urol 1998;159:654-7. http://dx.doi.org/10.1016/ S0022-5347(01)63694-2

18. Caudarella R, Vescini F, Buffa A, et al. Bone mass loss in calcium stone disease: Focus on hypercalciuria and metabolic factors. J Nephrol 2003;16:260-6.

19. Jehle S, Zanetti A, Muser J, et al. Partial neutralization of the acidogenic Western diet with potassium citrate increases bone mass in postmenopausal women with osteopenia. J Am Soc Nephrol 2006; 17:3213-22. http://dx.doi.org/10.1681/ASN.2006030233

20. Trinchieri A. Bone mineral content in calcium renal stone formers. Urol Res 2005;33:247-53. http:// dx.doi.org/10.1007/s00240-005-0498-y

21. Chung M, Lee J, Terasawa T, et al. Vitamin D with or without calcium supplementation for prevention of cancer and fractures: An updated meta-analysis for the U.S. Preventive Services Task Force. Ann Intern Med 2011;155:827-38. http://dx.doi.org/10.7326/0003-4819-155-12-201112200-00005

22. Jackson RD, LaCroix AZ, Gas $M$, et al. Calcium plus vitamin $D$ supplementation and the risk of fractures. N Engl J Med 2006;354:669-83. http://dx.doi.org/10.1056/NEJMo0055218

23. Siris ES, Chen YT, Abbott TA, et al. Bone mineral density thresholds for pharmaco-logical intervention to prevent fractures. Arch Intern Med 2004;164:1 108-12. http://dx.doi.org/10.1001/ archinte. 164.10 .1108

24. Sakhaee K, Maalouf NM, Kumar R, et al. Nephrolithiasis-associated bone disease: Pathogenesis and treatment options. Kidney Int 2011;79:393-403. http://dx.doi.org/10.1038/ki.2010.473

Correspondence: Dr. Sero Andonian, Assistant Professor of Urology, Royal Victoria Hospital, McGill University Health Centre, 687 avenue des Pins Ouest, Suite S6.92, Montreal, QC H3A 1A1; sero.andonian@muhc.mcgill.ca 